\title{
Association of Triglyceride-Glucose Index and the Presence of Sarcopenia in Type 2 Diabetes Patients
}

\section{Wenchao Hu}

Qingdao University

Dongming Xing ( $\nabla$ xdm_tsinghua@126.com )

Qingdao University https://orcid.org/0000-0003-2611-8042

\section{Research Article}

Keywords: triglyceride-glucose index, sarcopenia, skeletal muscle index

Posted Date: December 30th, 2021

DOI: https://doi.org/10.21203/rs.3.rs-1194442/v1

License: (c) (1) This work is licensed under a Creative Commons Attribution 4.0 International License. Read Full License 


\section{Abstract}

Objective: Triglyceride-glucose index (TyG index) has been used in healthy individuals as a marker of insulin resistance. Type 2 diabetes mellitus (T2DM) showed an increased risk of developing sarcopenia compared to control subjects. This study is performed to determine the association of TyG index with the presence of sarcopenia in T2DM patients.

Method: This study included 1098 T2DM patients who were recruited from the inpatients in Qilu Hospital (Qingdao). Skeletal muscle index (SMI) was measured using dual energy X-ray absorptiometry. Serum triglyceride (TG) and fasting plasma glucose (FPG) were measured and used to calculate TyG index.

Result: 119 male subjects (20.2\%) had sarcopenia, while 72 female subjects $(14.1 \%)$ had sarcopenia in T2DM patients. TyG index was correlated with a decreased risk of sarcopenia in both male and female T2DM groups. TyG index was found to be positively correlated with SMI after multivariate adjustment in male subjects. When TyG index was $\leq 9.5$, TyG index was positively correlated with SMI. However, when TyG index was $>9.5$, there was not a significant association between TyG index and SMI. Moreover, TyG index was not correlated with SMI after multivariate analysis in female subjects. However, TyG index was positively correlated with SMI when TyG index was $\leq 9$. When TyG index was $>9$, TyG index was negatively correlated with SMI, however, the correlation was not statistically significant.

Conclusion: TyG index is inversely correlated with the presence of sarcopenia in type 2 diabetes patients.

\section{Introduction}

Sarcopenia is characterized by a progressive and generalized loss of skeletal muscle mass and strength [1]. Sarcopenia is associated with an increased risk of falls and fractures, mobility disorders, disabilities, complications, infection, metabolic disorders, poor quality of life, and mortality [2]. Aging, physical inactivity, and malnutrition are considered as risk factors of sarcopenia [3]. Type 2 diabetes mellitus (T2DM) is one of the most widespread metabolic diseases. T2DM showed two to four-fold increased risk of developing sarcopenia compared with control subjects in Korea [4]. Veronese et al reported that the prevalence of sarcopenia was $28.4 \%$ in T2DM group while the prevalence of sarcopenia was $18.7 \%$ in the control group [5]. Considering the adverse effect of sarcopenia on the health, early detection and prevention of sarcopenia is important in T2DM patients. One potential way to achieve these goals is to identify possible risk factors or new biomarkers.

The triglyceride-glucose index (TyG index), the product of triglycerides (TG) and fasting plasma glucose (FPG), has been recommended as a reliable and simple surrogate index for insulin resistance [6]. A higher TyG-index has been significantly associated with fat distribution and fat depots [7], metabolic syndrome [8], cardiovascular disease [9], and diabetes [10]. We hypothesized that TyG-index may be correlated with sarcopenia. 
No previous studies focus on the relationship between TyG index and sarcopenia in T2DM patients. This study aims to investigate whether TyG index is associated with sarcopenia in T2DM patients.

\section{Materials And Methods}

\section{Patients}

This cross-sectional study was performed in a consecutive population of 1098 patients diagnosed with T2DM who were recruited from the inpatients in the Department of Endocrinology of Qilu hospital (Qingdao) from September of 2017 to September of 2019. T2DM was diagnosed according to the American Diabetic Association criteria with a fasting glucose level $\geq 7.0 \mathrm{mmol} / \mathrm{L}$ or 2 -hour postprandial plasma glucose level $\geq 11.1 \mathrm{mmol} / \mathrm{L}$. This study was approved by the Hospital ethics board and performed in compliance with the Declaration of Helsinki.

\section{The definition of sarcopenia}

Skeletal muscle index (SMI) was measured using dual energy X-ray absorptiometry (Hologic Discovery A, Waltham, MA, USA). Skeletal muscle index (SMI) was calculated as appendicular skeletal muscle mass in $\mathrm{kg}$ divided by height in meters squared. Sarcopenia was defined as SMI less than $7.0 \mathrm{~kg} / \mathrm{m}^{2}$ (in men) or $5.4 \mathrm{~kg} / \mathrm{m}^{2}$ (in women) [11].

\section{Measurements}

Anthropometric (height, weight and blood pressures), clinical, and laboratory analyses were performed. The information of duration of diabetes, history of smoking was collected. Body mass index (BMI) was calculated as weight in kilograms divided by height squared in meters $\left(\mathrm{kg} / \mathrm{m}^{2}\right)$. Blood was abstracted from all the subjects after an overnight fasting. FPG, total cholesterol (TC), TG, high-density lipoprotein cholesterol (HDL), and low-density lipoprotein cholesterol (LDL) were measured by a biochemistry automatic analyzer (Hitachi 7170, Tokyo, Japan). HbA1c levels were measured by high-performance liquid chromatography. TyG index was calculated using the following formula: In [fasting $\mathrm{TG}(\mathrm{mg} / \mathrm{dL}) \times$ FPG $(\mathrm{mg} / \mathrm{dL}) / 2]$.

\section{Statistical analysis}

All analyses were performed using the Statistical Package for the Social Sciences program (SPSS for Windows, version 22; Chicago, IL). Data are expressed as means \pm SD. Chi-square tests and unpaired $t$ test were utilized to determine the differences of characteristics between T2DM patients with and without sarcopenia. Logistic regression analysis was used to determine the risk factors for sarcopenia. Multiple linear regression analysis adjusted for potential confounding factors was used to further analyze the independent association between the TyG index and SMI. A smooth curve fitting was used to explore the relationship between the TyG index and SMI. Finally, a multivariate piecewise linear regression was 
further used to examine the threshold correlation of the TyG index and SMI according to the smooth curve fit.

\section{Results}

\section{The differences between subjects with and without sarcopenia}

As shown in Table 1, 119 male subjects $(20.2 \%)$ had sarcopenia, while 72 female subjects $(14.1 \%)$ had sarcopenia in T2DM patients. In male subjects, sarcopenia group showed higher age and HDL, as well as lower BMI, SMI, diastolic blood pressure (DBP), TG, and TyG index compared with non-sarcopenia group. In female subjects, T2DM patients with sarcopenia showed higher age, as well as lower BMI, SMI, TG, and TyG index compared with those without sarcopenia.

\section{The association between the TyG index and sarcopenia}

The association between the clinical characteristics and sarcopenia was shown in Table 2. TyG index was correlated with a decreased risk of sarcopenia in both male and female T2DM groups. After adjusting for age, TyG index was inversely associated with sarcopenia in both male and female T2DM patients. In addition, TyG index was still inversely correlated with sarcopenia after adjusting for age, smoking, SBP, DBP, and duration of diabetes.

\section{The association between SMI and other characteristics}

As shown in Table 3, SMI was correlated with age, duration of diabetes, BMI, DBP, TG, HDL, and TyG index in male subjects. In female subjects, SMI was correlated with age, BMI, DBP, HDL, and TyG index.

\section{Non-linear association between TyG index and SMI}

As shown in Figure 1, there might be some non-linear association between TyG index and SMI in male subjects. We further applied a two-piecewise linear regression model (Table 4). TyG index was found to be non-linearly correlated with SMI after multivariate adjustment for age, smoking, SBP, DBP, and duration of diabetes. When TyG index was $\leq 9.5$, TyG index was positively correlated with SMI. However, when TyG index was $>9.5$, there was not a significant association between TyG index and SMI.

As shown in Figure 2, there might be some non-linear association between TyG index and SMI in female subjects. We further applied a two-piecewise linear regression model (Table 5). TyG index was not correlated with SMI after adjusting for age, smoking, SBP, DBP, and duration of diabetes. However, when TyG index was $\leq 9$, TyG index was positively correlated with SMI. When TyG index was $>9$, TyG index was negatively correlated with SMI, however, the correlation was not statistically significant.

\section{Discussion}


The present study indicates that TyG index is correlated with a decreased risk of sarcopenia in T2DM patients. TyG index was found to be positively correlated with SMI after multivariate adjustment in male subjects. When TyG index was $<9$, TyG index was positively correlated with SMI in female subjects.

Obesity was considered as a traditional risk factor for sarcopenia. Kadoma Sarcopenia Study performed in Japan showed that obesity and hypertension were independent predictors of sarcopenia [12]. The metabolic risk factors including BMI were significantly related to loss of skeletal muscle and sarcopenia [13]. However, other studies got the reverse results. Low BMI is a predictive risk factor of sarcopenia among adults living in nursing homes [14]. A longitudinal Study in China with 4 years of follow-up demonstrated that a high BMI was protective against the decline in muscle mass and sarcopenia, but represented a risk factor for low gait speed [15]. As in T2DM patients, most studies indicated that BMI was negatively correlated with sarcopenia. Lower BMI, female gender, and aging were risk factors for sarcopenia in elderly T2DM [16]. The prevalence of sarcopenia significantly decreases as BMI increases in T2DM patients [17] [18] [19]. The similar results were found in our study. The present study also showed that BMI is significantly lower in T2DM individuals with sarcopenia compared with those without sarcopenia.

The present study indicated that sarcopenia group showed lower FPG than non-sarcopenia group in T2DM patients; however, the difference was not statistically significant. Other investigators also got the similar results [20] [21] [22]. Previous results have demonstrated that TG was significantly lower in T2DM patients with sarcopenia compared with those without sarcopenia [16] [18] [21] [23]. Our investigation also showed that sarcopenia group showed significantly decreased TG than non-sarcopenia group in T2DM patients. This may be contradictory with our traditional opinion. Obesity, higher glucose, and hyperlipidemia are correlated with a higher risk of developing diabetic complication and cardiovascular disease. However, as far as the condition of sarcopenia concerned, obesity, higher glucose, and hyperlipidemia are correlated with a decreased risk of developing sarcopenia in T2DM patients.

This study has several potential limitations. First, the conclusion is limited by relatively small sample size. Secondly, the cross-sectional nature of the data limited the strength of conclusion. The causative relation must be confirmed by future longitudinal studies.

In conclusion, TyG index is inversely correlated with the presence of sarcopenia in type 2 diabetes patients.

\section{Declarations}

\section{Funding Statement}

No funding.

\section{Conflict of Interest Statement}

The authors declare that there is no conflict of interest regarding the publication of this article. 


\section{Ethics approval}

This study was approved by the ethics board of Qilu Hospital of Shandong University (Qingdao).

\section{Data availability statement}

Data are available upon reasonable request.

\section{Acknowledgments}

No acknowledgments.

\section{References}

1. Umegaki H. Sarcopenia and frailty in older patients with diabetes mellitus. Geriatr Gerontol Int. 2016;16:293-9.

2. Hanna JS. Sarcopenia and critical illness: a deadly combination in the elderly. JPEN J Parenter Enteral Nutr. 2015;39:273-81.

3. Simsek H, Meseri R, Sahin S, et al. Prevalence of sarcopenia and related factors in communitydwelling elderly individuals. Saudi Med J. 2019;40:568-74.

4. Park SW, Goodpaster BH, Lee JS, et al. Excessive loss of skeletal muscle mass in older adults with type 2 diabetes. Diabetes Care. 2009;32:1993-7.

5. Veronese N, Stubbs B, Punzi L, et al. Effect of nutritional supplementations on physical performance and muscle strength parameters in older people: A systematic review and meta-analysis. Ageing Res Rev. 2019;51:48-54.

6. Simental-Mendía LE, Rodríguez-Morán M, Guerrero-Romero F. The product of fasting glucose and triglycerides as surrogate for identifying insulin resistance in apparently healthy subjects. Metab Syndr Relat Disord. 2008;6:299-304.

7. Aras Ş, Üstünsoy S, Armutçu F. Indices of Central and Peripheral Obesity; Anthropometric Measurements and Laboratory Parameters of Metabolic Syndrome and Thyroid Function. Balkan Med J. 2015;32:414-20.

8. Angoorani P, Heshmat R, Ejtahed HS, et al. Validity of triglyceride-glucose index as an indicator for metabolic syndrome in children and adolescents: the CASPIAN-V study. Eat Weight Disord. 2018;23:877-83.

9. Sánchez-Î́nigo L, Navarro-González D, Fernández-Montero A, Pastrana-Delgado J, Martínez JA. The TyG index may predict the development of cardiovascular events. Eur J Clin Invest. 2016;46:189-97.

10. Navarro-González D, Sánchez-Íñigo L, Fernández-Montero A, Pastrana-Delgado J, Martinez JA. TyG Index Change Is More Determinant for Forecasting Type 2 Diabetes Onset Than Weight Gain. Med (Baltim). 2016;95:e3646. 
11. Chen LK, Liu LK, Woo J, et al. Sarcopenia in Asia: consensus report of the Asian Working Group for Sarcopenia. J Am Med Dir Assoc. 2014;15:95-101.

12. Kurose S, Nishikawa S, Nagaoka T, et al. Prevalence and risk factors of sarcopenia in communitydwelling older adults visiting regional medical institutions from the Kadoma Sarcopenia Study. Sci Rep. 2020;10:19129.

13. Du Y, Oh C, No J. Associations between Sarcopenia and Metabolic Risk Factors: A Systematic Review and Meta-Analysis. J Obes Metab Syndr. 2018;27:175-85.

14. Senior HE, Henwood TR, Beller EM, Mitchell GK, Keogh JW. Prevalence and risk factors of sarcopenia among adults living in nursing homes. Maturitas. 2015;82:418-23.

15. Zhang Y, Chen X, Hou L, et al. Prevalence and Risk Factors Governing the Loss of Muscle Function in Elderly Sarcopenia Patients: A longitudinal Study in China with 4 Years of Follow-Up. J Nutr Health Aging. 2020;24:518-24.

16. Chen F, Xu S, Wang Y, et al. Risk Factors for Sarcopenia in the Elderly with Type 2 Diabetes Mellitus and the Effect of Metformin. J Diabetes Res. 2020;2020:3950404.

17. Sazlina SG, Lee PY, Chan YM, Hamid A, Tan MS. NC. The prevalence and factors associated with sarcopenia among community living elderly with type 2 diabetes mellitus in primary care clinics in Malaysia. PLoS One. 2020;15(5):e0233299.

18. Bouchi R, Fukuda T, Takeuchi T, Minami I, Yoshimoto T, Ogawa Y. Sarcopenia is associated with incident albuminuria in patients with type 2 diabetes: A retrospective observational study. J Diabetes Investig. 2017;8:783-7.

19. Hashimoto Y, Kaji A, Sakai R, et al. Sarcopenia is associated with blood pressure variability in older patients with type 2 diabetes: A cross-sectional study of the KAMOGAWA-DM cohort study. Geriatr Gerontol Int. 2018;18:1345-9.

20. Okamura T, Hashimoto Y, Miki A, et al. High brain natriuretic peptide is associated with sarcopenia in patients with type 2 diabetes: a cross-sectional study of KAMOGAWA-DM cohort study. Endocr J. 2019;66:369-77.

21. de Freitas MM, de Oliveira VLP, Grassi T, et al. Difference in sarcopenia prevalence and associated factors according to 2010 and 2018 European consensus (EWGSOP) in elderly patients with type 2 diabetes mellitus. Exp Gerontol. 2020;132:110835.

22. Okamura T, Miki A, Hashimoto $Y$, et al. Shortage of energy intake rather than protein intake is associated with sarcopenia in elderly patients with type 2 diabetes: A cross-sectional study of the KAMOGAWA-DM cohort. J Diabetes. 2019;11:477-83.

23. Sung MJ, Lim TS, Jeon MY, et al. Sarcopenia Is Independently Associated with the Degree of Liver Fibrosis in Patients with Type 2 Diabetes Mellitus. Gut Liver. 2020;14:626-35.

\section{Tables}

Table 1 The characteristic differences between T2DM patients with and without sarcopenia 


\begin{tabular}{|c|c|c|c|c|c|c|}
\hline \multirow[b]{2}{*}{ icteristics } & \multicolumn{3}{|c|}{ Male $(\mathrm{n}=588)$} & \multicolumn{3}{|c|}{ Female $(n=510)$} \\
\hline & $\begin{array}{l}\text { Non-sarcopenia } \\
\qquad(\mathrm{n}=469)\end{array}$ & $\begin{array}{l}\text { Sarcopenia } \\
\qquad(\mathrm{n}=119)\end{array}$ & $P$ value & $\begin{array}{l}\text { Non-sarcopenia } \\
\qquad(\mathrm{n}=438)\end{array}$ & $\begin{array}{l}\text { Sarcopenia } \\
\qquad(\mathrm{n}=72)\end{array}$ & $P$ value \\
\hline tears) & $54.64 \pm 11.95$ & $60.67 \pm 13.45$ & $<0.001$ & $61.1 \pm 10.88$ & $65.47 \pm 11.28$ & 0.002 \\
\hline ing & & & 0.615 & & & 0.99 \\
\hline$(\mathrm{n}, \%)$ & 217 & 252 & & 432 & 6 & \\
\hline$(\mathrm{n}, \%)$ & 52 & 67 & & 71 & 1 & \\
\hline ion (years) & $8.02 \pm 6.66$ & $9.03 \pm 5.83$ & 0.133 & $8.73 \pm 5.98$ & $9.17 \pm 6.73$ & 0.574 \\
\hline $\left.\mathrm{kg} / \mathrm{m}^{2}\right)$ & $27.57 \pm 4.09$ & $23.65 \pm 4.83$ & $<0.001$ & $27.33 \pm 4.58$ & $22.42 \pm 2.98$ & $<0.001$ \\
\hline & $8.18 \pm 1.5$ & $6.45 \pm 0.48$ & $<0.001$ & $6.5 \pm 0.75$ & $5.07 \pm 0.33$ & $<0.001$ \\
\hline mmHg) & $140.44 \pm 19.71$ & $137.5 \pm 21.34$ & 0.155 & $143.38 \pm 20.71$ & $143.07 \pm 21.95$ & 0.906 \\
\hline mmHg) & $82.54 \pm 12.89$ & $78.66 \pm 13.02$ & 0.004 & $76.62 \pm 12.73$ & $76.58 \pm 11.14$ & 0.982 \\
\hline $\mathrm{mol} / \mathrm{L})$ & $2.3 \pm 2.25$ & $1.41 \pm 0.81$ & $<0.001$ & $1.85 \pm 1.4$ & $1.44 \pm 0.79$ & 0.016 \\
\hline $\mathrm{mol} / \mathrm{L})$ & $4.52 \pm 1.16$ & $4.47 \pm 1.09$ & 0.683 & $4.7 \pm 1.35$ & $4.6 \pm 1.16$ & 0.557 \\
\hline $\mathrm{mmol} / \mathrm{L})$ & $2.98 \pm 0.91$ & $2.97 \pm 0.94$ & 0.91 & $3 \pm 0.93$ & $2.89 \pm 0.98$ & 0.37 \\
\hline $\mathrm{mmol} / \mathrm{L})$ & $1.14 \pm 0.27$ & $1.23 \pm 0.35$ & 0.001 & $1.29 \pm 0.37$ & $1.36 \pm 0.35$ & 0.173 \\
\hline mmol/L) & $8.15 \pm 2.77$ & $7.97 \pm 3.17$ & 0.535 & $7.74 \pm 2.9$ & $7.04 \pm 2.76$ & 0.057 \\
\hline c $(\%)$ & $8.43 \pm 2.09$ & $8.21 \pm 2.18$ & 0.35 & $8.31 \pm 2.02$ & $8.15 \pm 2.13$ & 0.566 \\
\hline ıdex & $9.28 \pm 0.84$ & $8.88 \pm 0.76$ & $<0.001$ & $9.1 \pm 0.76$ & $8.81 \pm 0.7$ & 0.002 \\
\hline
\end{tabular}

Table 2 The association between TyG and sarcopenia

\begin{tabular}{l|c|c|c|c|c|c|}
\hline & \multicolumn{3}{|c|}{ Male } & \multicolumn{3}{c|}{ Female } \\
\hline del & Unjusted model & Model 2 & Model 3 & Unjusted model & Model 2 & Model 3 \\
\hline R & $0.526(0.401-$ & 0.601 & 0.612 & $0.576(0.404-$ & 0.602 & 0.592 \\
CI) & $0.691)$ & $(0.454-$ & $(0.457-$ & $0.823)$ & $(0.418-$ & $(0.408-$ \\
& & $0.797)$ & $0.819)$ & & $0.868)$ & $0.858)$ \\
\hline lue & $<0.001$ & $<0.001$ & 0.001 & 0.002 & 0.007 & 0.006 \\
\hline
\end{tabular}

model 1 was not adjusted;

model 2 was adjusted for age;

model 3 was adjusted for age, smoking, SBP, DBP, duration of diabetes

Table 3 The association between TyG and SMI 


\begin{tabular}{|c|c|c|c|c|}
\hline & \multicolumn{2}{|l|}{ Male } & \multicolumn{2}{|l|}{ Female } \\
\hline & $\beta(95 \% \mathrm{CI})$ & $P$ value & $\beta(95 \% \mathrm{CI})$ & $P$ value \\
\hline Age (years) & $-0.225(-0.037,-0.018)$ & $<0.001$ & $-0.231(-0.028,-0.013)$ & $<0.001$ \\
\hline Smoking & $0.017(-0.196,0.3)$ & 0.68 & $0.038(-0.407,1.049)$ & 0.387 \\
\hline Juration (years) & $-0.092(-0.04,-0.003)$ & 0.026 & $-0.014(-0.016,0.012)$ & 0.751 \\
\hline $3 \mathrm{MI}\left(\mathrm{kg} / \mathrm{m}^{2}\right)$ & $0.152(0.128,0.177)$ & $<0.001$ & $0.125(-0.11,0.139)$ & $<0.001$ \\
\hline $\begin{array}{l}\text { ¡BP (mmHg) } \\
\text { JBP (mmHg) }\end{array}$ & $0.005(-0.001,0.011)$ & 0.114 & $0.003(-0.001,0.007)$ & 0.213 \\
\hline$\Gamma \mathrm{G}(\mathrm{mmol} / \mathrm{L})$ & $0.017(0.007,0.026)$ & 0.001 & $0.01(0.003,0.017)$ & 0.003 \\
\hline ГС $(\mathrm{mmol} / \mathrm{L})$ & $0.115(0.025,0.143)$ & 0.005 & $0.069(-0.013,0.114)$ & 0.118 \\
\hline $\mathrm{LDL}(\mathrm{mmol} / \mathrm{L})$ & $0.02(-0.082,0.135)$ & 0.629 & $-0.01(-0.072,0.056)$ & 0.814 \\
\hline $\mathrm{FDL}(\mathrm{mmol} / \mathrm{L})$ & $0.02(-0.102,0.168)$ & 0.631 & $0.004(-0.086,0.094)$ & 0.933 \\
\hline FPG (mmol/L) & $-0.55(-0.976,-0.123)$ & 0.012 & $-0.266(-0.497,-0.035)$ & 0.024 \\
\hline HbA1c (\%) & $0.027(-0.029,0.058)$ & 0.514 & $-0.001(-0.03,0.029)$ & 0.989 \\
\hline TyG index & $-0.035(-0.132,0.009)$ & 0.225 & $-0.014(-0.057,0.029)$ & 0.521 \\
\hline & $0.298(0.153,0.443)$ & $<0.001$ & $0.123(0.012,0.233)$ & 0.03 \\
\hline
\end{tabular}

Table 4 The correlation between TyG and SMI in male subjects.

\begin{tabular}{|c|c|c|}
\hline Models & $\beta(95 \%$ CI $)$ & $P$ value \\
\hline Model I & & \\
\hline One line slope & $0.094(0.018,0.324)$ & 0.029 \\
\hline Model II & & \\
\hline Turning point & 9.5 & \\
\hline$<9.5$ slope 1 & $0.121(0.059,0.757)$ & 0.022 \\
\hline$>9.5$ slope 2 & $0.061(-0.149,0.393)$ & 0.375 \\
\hline
\end{tabular}

The independent association between the TyG index and SMI.

Model I, linear analysis; Model II, non-linear analysis. Adjustment variables: age, smoking, SBP, DBP, duration of diabetes.

Table 5 The correlation between TyG and SMI in female subjects. 


\begin{tabular}{|c|c|c|}
\hline Models & $\beta(95 \%$ CI $)$ & $P$ value \\
\hline Model I & & \\
\hline One line slope & $0.061(-0.032,0.186)$ & 0.165 \\
\hline Model II & & \\
\hline Turning point & 9 & \\
\hline$<9$ slope 1 & $0.18(0.154,0.804)$ & 0.004 \\
\hline$>9$ slope 2 & $-0.051(-0.311,0.13)$ & 0.42 \\
\hline
\end{tabular}

The independent association between the TyG index and SMI.

Model I, linear analysis; Model II, non-linear analysis. Adjustment variables: age, smoking, SBP, DBP, duration of diabetes.

\section{Figures}




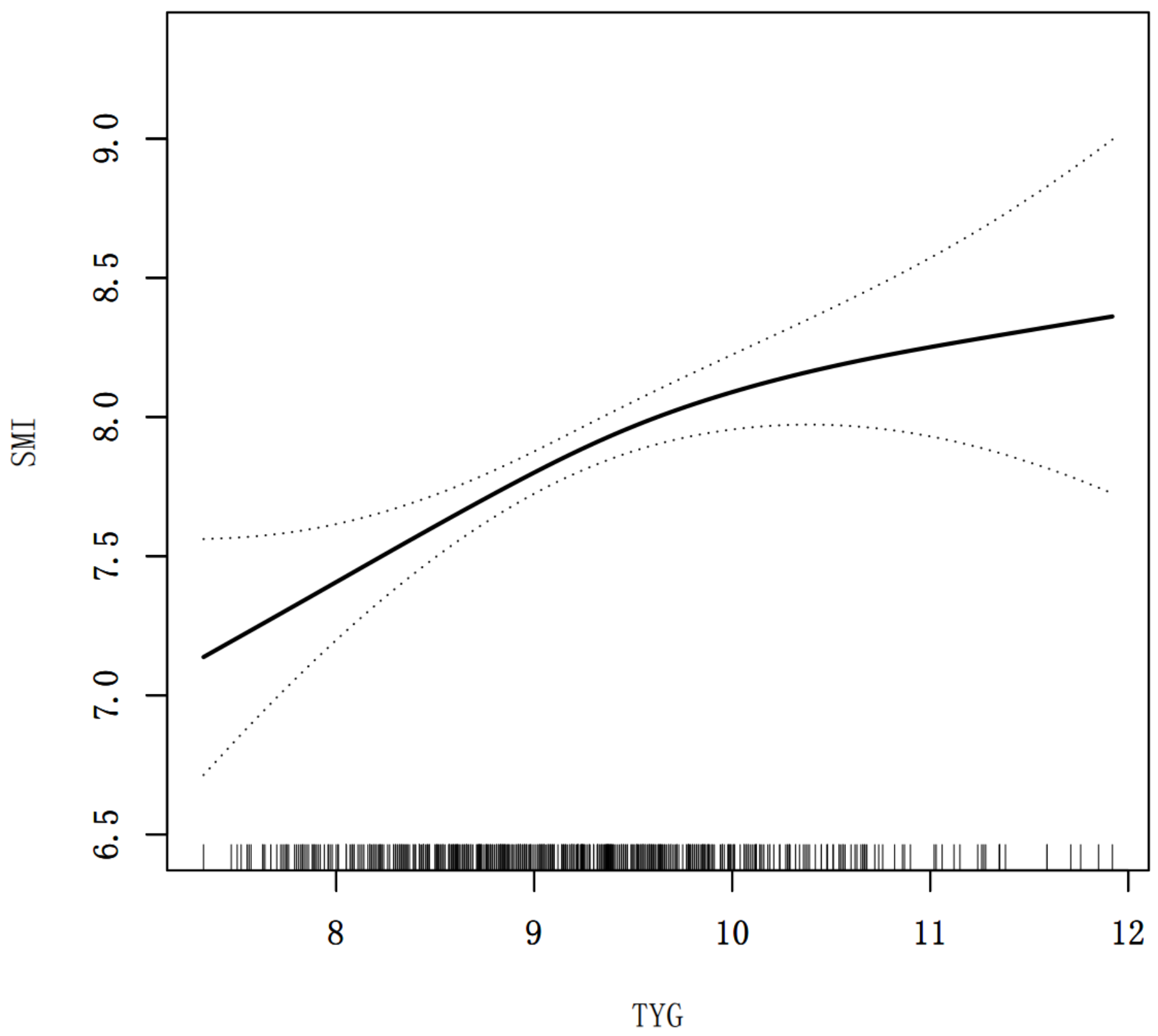

Figure 1

The relationship between TyG index and SMI by smooth curve fitting in male subjects. 


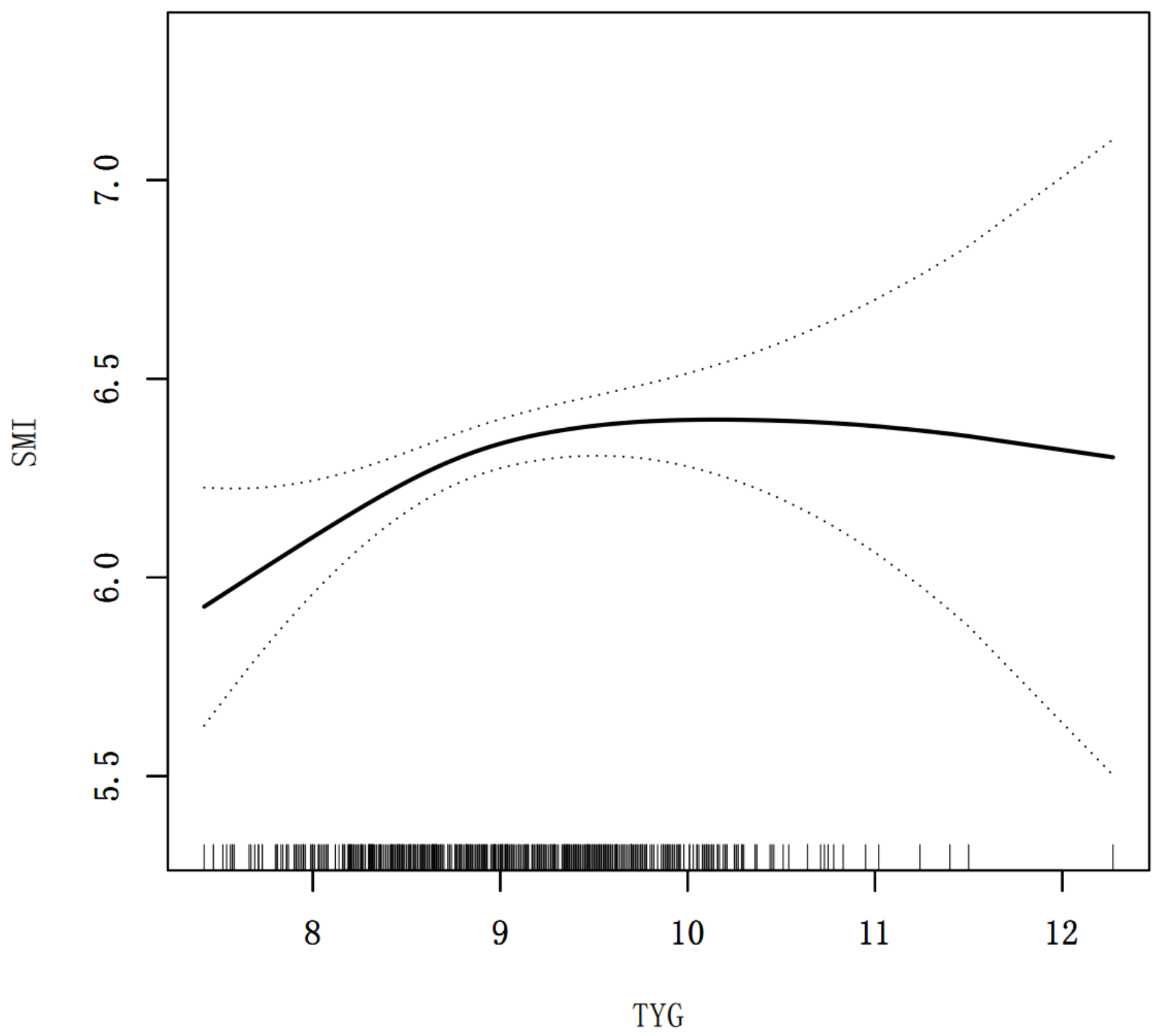

Figure 2

The relationship between TyG index and SMI by smooth curve fitting in female subjects. 